\title{
Resolution Achievement of 40.5 pm in Scanning Transmission Electron Microscopy using 300 kV Microscope with Delta Corrector
}

\author{
Shigeyuki Morishita ${ }^{1}$, Ryo Ishikawa ${ }^{2}$, Yuji Kohno ${ }^{1}$, Hidetaka Sawada ${ }^{1}$, Naoya Shibata ${ }^{2}$ and Yuichi \\ Ikuhara $^{2}$ \\ 1. JEOL Ltd, Akishima, Tokyo, Japan. \\ 2. Institute of Engineering Innovation, The University of Tokyo, Bunkyo-ku, Tokyo, Japan.
}

Due to the recent development of aberration correctors, a spatial resolution of transmission electron microscopy (TEM) and scanning TEM (STEM) has been improved. With the correctors, sub-angstrom resolution has become common and several resolution achievements better than 50 pm have been reported. The best resolution reported so far was 45 pm in STEM (Si [114]) [1] and 44 pm in TEM (GaN [411], $43 \mathrm{pm}$ by lattice fringe of $\mathrm{W}$ ) [2]. These resolutions have been used for determining atomic positions of many structures. To increase the precision of the atomic identification, continual improvement of the resolution is naturally desirable. On top of that, it is requested for the analyses of an electric field of each atom, which were accomplished by using differential phase contrast imaging. In this paper, we report the improvement of the spatial resolution using a $300 \mathrm{kV}$ aberration corrected STEM.

When a microscope is under the free-aberration condition, the resolution is limited by the diffraction limit, which is expressed as $0.61 \lambda / \alpha$, where $\lambda$ and $\alpha$ are wavelength and convergence semi-angle, respectively. The geometric aberration correction realizes a constant phase of incident beam over a large convergence angle. This enabled us to use a large convergence angle for STEM imaging, which results in the improvement of the diffraction limit. However, since there is a residual six-fold astigmatism, the aberration-corrected angle has been limited up to approximately $30 \mathrm{mrad}$ in a microscope with a standard double-hexapole type corrector. To increase the convergence angle, the higher-order aberration corrector is required. For this purpose, we have developed a delta corrector for a probe-forming system. The delta-type corrector can compensate geometrical aberrations up to fifth order including the six-fold astigmatism, which is originally designed for a low-voltage atomic-resolution microscope [3]. In this study, we have newly designed the delta corrector for the higher voltage of $300 \mathrm{kV}$, and it has been installed to GRAND ARM (JEM-ARM300F) in the University of Tokyo, as shown in Fig. 1a. With this microscope, a flat phase area in a Ronchigram was extended to be $70 \mathrm{mrad}$ at $300 \mathrm{kV}$ (Fig. 1b). The dominant aberration was sixth-order three-lobe aberration [4], which was measured to be $1.8 \mathrm{~mm}$ by using a segmental Ronchigram autocorrelation function matrix method. The measurement also indicated that the coefficients of third-, fourth-, and fifth-order aberrations are less than $0.5,15$ and $50 \mu \mathrm{m}$, respectively. This large flat phase area was contributed to the reduction of diffraction limit.

For the demonstration of resolution enhancement in atomic resolution STEM imaging, we obtained the high-angle annular dark field (HAADF) images of GaN thin film observed along the [212] direction at $300 \mathrm{kV}$. To investigate the diffraction limit, we obtained images with different convergence angles from 20 to $40 \mathrm{mrad}$. Figures 2a-c show the HAADF images of GaN viewed along the [212] and intensity profiles. The images were taken with a dwell time of $38 \mu$ s per pixel and averaged for 10 raw images. The detection inner angle was $55 \mathrm{mrad}$. Because of the excessive contrast by Z-number in the HAADF images, only $\mathrm{Ga}$ atomic columns are visible. The image at $40 \mathrm{mrad}$ shows clearly resolved Ga-Ga atomic dumbbells, which corresponds to a separation of $40.5 \mathrm{pm}$, though the image at $20 \mathrm{mrad}$ shows 
unresolved Ga-Ga dumbbells. The intensity profile for $40 \mathrm{mrad}$ shows the clear dip between $\mathrm{Ga}$ atoms, whose contrast is $13 \%$. Figure $2 \mathrm{~d}$ shows the modulus of Fourier transform of the image at the convergence semi-angle of $40 \mathrm{mrad}$. The power spectrum indicates the $60 \overline{6}$ spot corresponding to $(40.5$ $\mathrm{pm})^{-1}$. In conclusion, we demonstrated the spatial resolution of $40.5 \mathrm{pm}$ by developed $300 \mathrm{kV}$ STEM with the fifth-order geometrical aberration corrector [5]. We believe that this high-resolution STEM will be used for various precise analyses of various materials.

\section{References:}

[1] H Sawada et al, Microscopy 64 (2015), p. 213.

[2] T Akashi et al, Appl. Phys. Lett. 106 (2015), p. 074101.

[3] H Sawada et al, J. Electron Microsc. 58 (2009), p. 341.

[4] S Morishita et al, Microscopy (2018).

[5] S Morishita et al, Microscopy 67 (2017), p. 46.
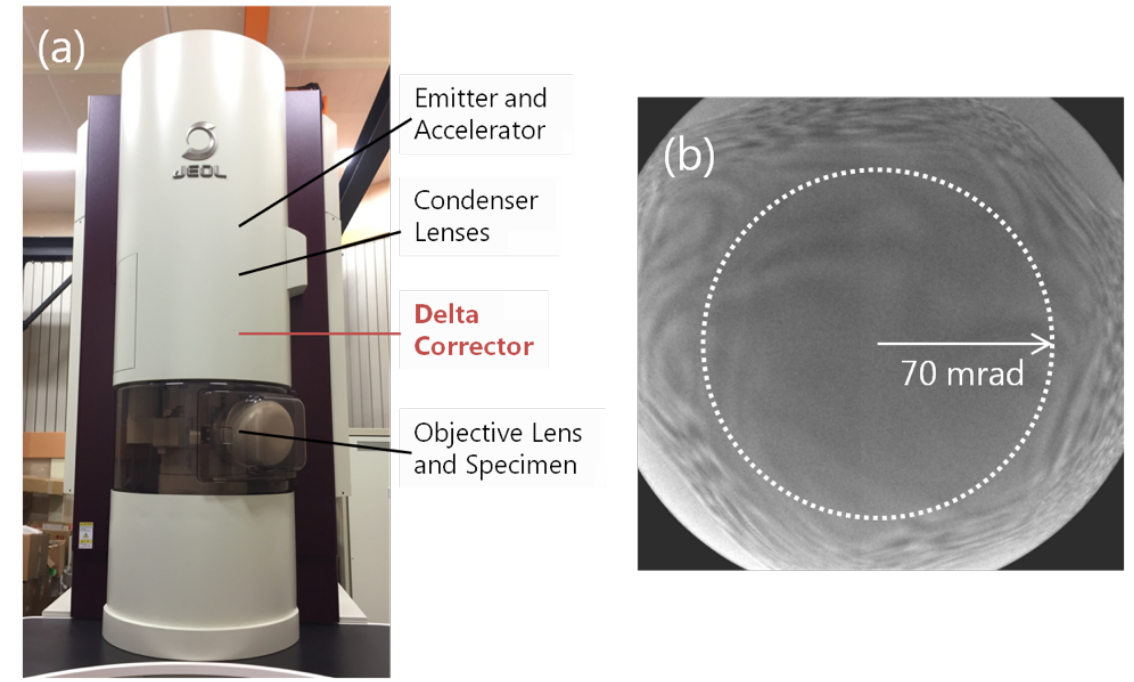

Figure 1. (a) $300 \mathrm{kV}$ electron microscope (GRAND ARM) equipped with a delta corrector and a cold field emission gun for STEM. (b) Ronchigram at $300 \mathrm{kV}$ after aberration correction with delta type corrector for probe forming system.
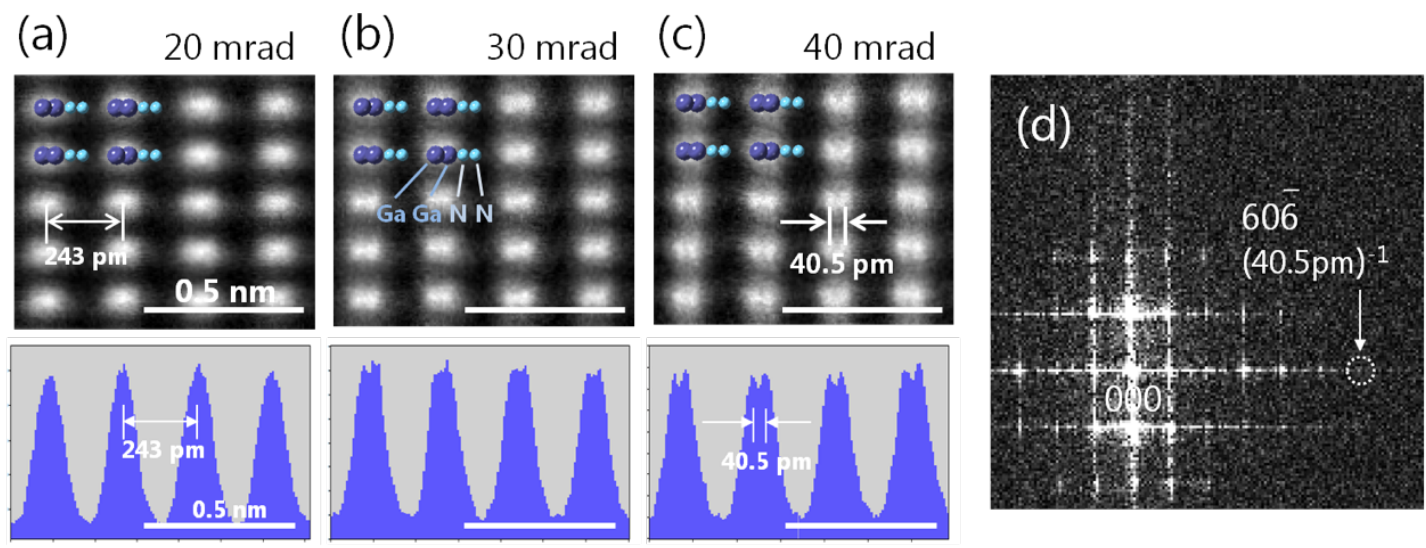

Figure 2. ADF STEM images of GaN [212] with convergence semi-angles of (a) 20 mard, (b) 30 mrad and (c) $40 \mathrm{mrad}$. Intensity profiles of these images are also shown below. (d) Modulus of the Fourier transform from the image obtained with the $40 \mathrm{mrad}$ convergence angle. 